\title{
Design and Implementation of Tourist Landmarks Recommending System
}

\author{
Shengfu Zou ${ }^{1, a}$, Chuan $\mathrm{Guo}^{2, \mathrm{~b}}$, Kaixuan $\mathrm{Ji}^{3, \mathrm{c}}$, Yang Gao ${ }^{4, \mathrm{~d}}$, and Hongwei \\ Zhao ${ }^{5, \mathrm{e}}$ \\ ${ }^{1,2,3,4,5}$ College of Software Engineering, Jilin University, Changchun 130012, China \\ aemail:zsf657135507@163.com \\ bemail:15567740960@163.com \\ cemail:18844546502@qq.com \\ demail:w2028953318@sina.com
}

Corresponding author: zhaohw@jlu.edu.cn

Keywords: MVC, B/S structure, JSP, Image Retrieval, Landmarks Recommending.

\begin{abstract}
This paper presents the design and implementation of a tourist landmarks recommending system, which adopts the content-based image retrieval technology. The traditional text-based image retrieval technology has been hardly satisfying the users' requirements. To lower the cost, this system uses B/S architecture, distributing the work of logical transaction operation to the server side. Meanwhile, for promoting the maintainability and reducing the coupling of code, this system uses MVC model to organize code. Also, the users upload the data as input. The refined multiple-feature fusion algorithm and bidirectional matching strategy enable the functions like landmarks recognition or searching similar landmarks, which satisfy the customers' need preferably.
\end{abstract}

\section{Introduction}

Recent years, as the rapid development of Internet technology and tourism, the relevant pictures and their information become more and more valuable and indispensable. How to mine and obtain the information from those pictures is always a hot pot in data mining, image processing and also related academic fields [1-3].

The existed landmarks retrieval systems almost rely on text-based image retrieval technology (TBIR): firstly building the text information collection and then searching the text information related to the pictures. But in this way, the user is required to have clear and accurate text illustration of the pictures they want to search. Moreover, although the users know what they want and have definite key words, the same words could be ambiguous to the text-based system. As a result, the outputted landmarks may differ much from what the users understand.

On the other hand, content-based image retrieval (CBIR) tries out solving those problems. CBIR utilizes the characteristics of the images properly; it process according to the features of image like the color, texture, SIFT, instead of the text information of pictures [4]. A picture contains lots of valuable information, which sometimes couldn't be expressed by key words comprehensively. Therefore, it's meaningful for both enterprise and individual to obtain accurate recommending information through inputting related pictures. However, most present landmark retrieval systems don't support this technology; even support, the result is also unsatisfactory. So, it's necessary to apply the CBIR to landmarks recommending fields [5].

\section{Requirement Analysis}

\subsection{Users' Requirements}

This system is aimed at people who are interested in traveling. And this system has those functions: landmarks recognition, recommending similar landmarks, evaluating landmarks. Meanwhile, this system should be extendable and maintainable. 
(1) Landmarks recognition: according to the input pictures or the link of pictures, if possible, the system could recognize the landmarks in the pictures, and then provide relevant information and dynamics.

(2) Recommending similar landmarks: according to the input pictures or the link of pictures, the system could recommend related or similar landmarks with the pictures.

(3) Evaluating landmarks: the users could evaluate the landmarks according to real circumstances, which could provide valuable reference information.

(4) System extension: through second-hand development, the system could extend with new functions, for adapting the future demand.

\subsection{Functional Requirements}

This system includes the following three aspects on functional requirements.

(1) Building landmarks information resource database: Collecting the information related to the landmarks. For example, introduction, history, price of tickets, or the stories about this landmark. The resource database could provide data to the retrieval process.

(2) Building image retrieval subsystem: through the image information the user uploads, the system utilizes the image retrieval algorithm and then calculates the similarity between the landmarks in the database and the input picture.

(3) Building user evaluating subsystem: the evaluation is shared to all the users, as reference information to the landmarks.

\section{System's Integral Design}

To improve the reusability of the code and the quality of the software, and also consider the aiming circumstance and the generality of the functions, this system uses the B/S framework and MVC. JSP and MySQL database are used for software development.

\subsection{System's Structure}

This system could be divided into four parts: browser, WeChat public platform, web server, MySQL database. The organization of the four parts is as Figure 1.

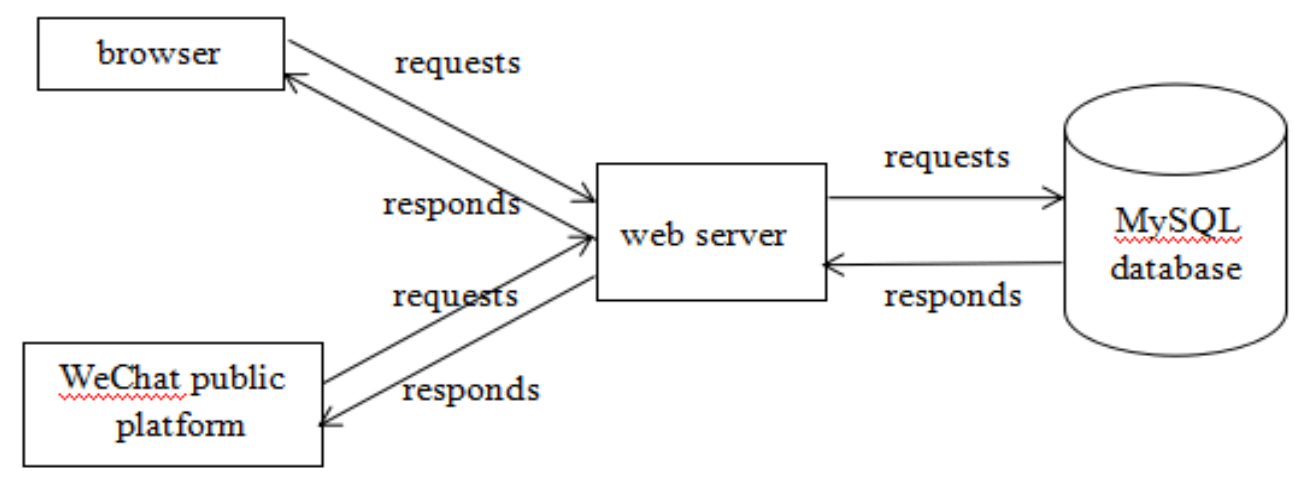

Figure 1.System’s structure diagram.

Browser and WeChat public platform: mainly the display of the system. The users send the requests, such as recognizing the landmark, or recommending similar landmarks through the browser or the WeChat public platform. Then the server responds the requests and displays the result on the electrical device.

Web Server: mainly transaction of the system. The server receives the requests from the users, and processes the retrieval algorithms, then connects and submits data operation application to the Web database. The database returns the operated data to the Web Server and then to the browser or the WeChat public platform.

MySQL database: system's data. The database receives the requests - mainly the application about image query or similarity sorting from the Web server. Then the database returns the results to the Web server. 


\subsection{System's Technical Architecture}

According to the standard of MVC model, this system could be divided into three parts: model layer, view layer, and control layer.

Model layer: In order to provide unified data for result display of view layer, numbers of classes are involved in this layer; they are used to encapsulate the data from MySQL database.

View layer: this layer collects the request information from the users and transmits the information to the control layer. After control layer returning data, view layer display the results to the users.

Control layer: this layer receives the request information from the view layer, combine the request information and the data from MySQL database, and then calculate the data through the refined algorithm and return the information the user need to view layer.

\section{Implementation of System's Functions}

\subsection{Build the Landmarks’ Information Resource Database}

After analyzing the data involved in this system, we build up 10 database tables to memory the fundamental information of the landmarks. For example, table color_desc is used to store the RGB color feature of each picture, of which the feature is presented by 256-dimension feature vectors. And table AttractionBackup documents the basic information and the dynamics of the landmarks which related to the pictures, such as the name, introductions, phone number of the landmarks. Table Values is used to memory the evaluation of the landmarks from the users.

Afterwards, we utilize the tech "web crawler", collect the pictures and the basic information of landmarks from Internet, and extract the image feature from the pictures, and then insert the feature data into the database. Meanwhile, we insert the basic information of the landmarks into corresponding tables. For the pictures we attain, we would upload the pictures on the server and document the links of the pictures in the database.

\subsection{Build the Image Retrieval Subsystem}

At first, html and css tech are used to program the input interface, the completed interface is as Figure 2. This interface mainly display this system's logo "sosight”; meanwhile, php tech complete the function of picture upload, to commit the input picture to the servlet program, achieving two upload approaches: local picture and picture links.

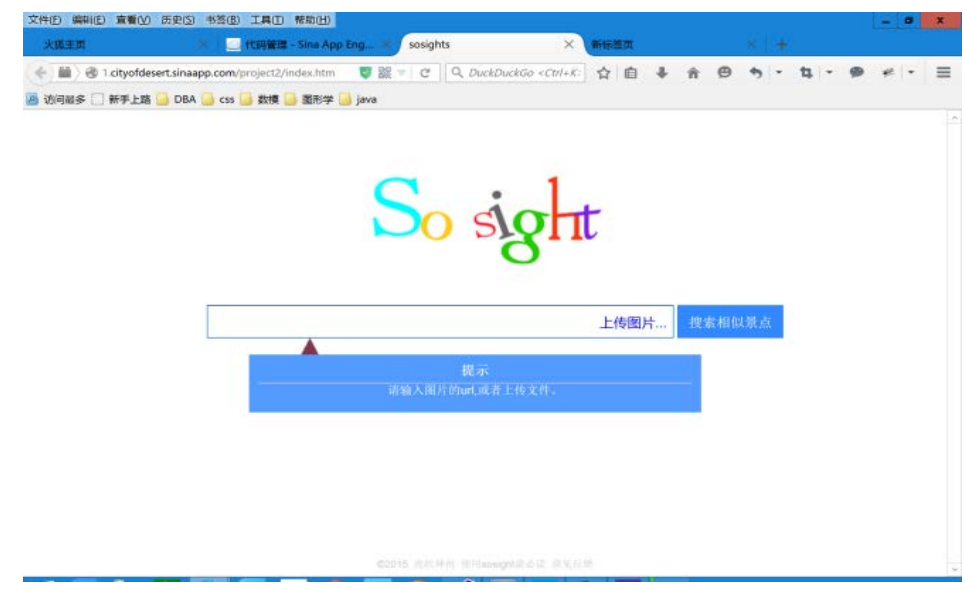

Figure 2 Upload picture interface

Then, we program the matlab functions which could extract the SIFT feature and color feature and then generalize these features. The functions are invoked for feature extraction in the servlet. While matching the pictures, the system adopts the multiple feature fusion algorithm and bidirectional matching strategy. This system first matches the pictures in the data resource with the input picture according to the color feature. Based on the picture sets of the color feature matching, we process the SIFT feature matching, which is matching the SIFT feature of the input pictures with SIFT feature in the data resource. After two times of matching, we basically attain the similarity of 
the resource pictures with the input picture. And then the system displays the landmarks of these pictures and the relevant information of these landmarks.

Finally, we program the result display interface, to show the retrieval results above on the browser. As figure 3, while implementing displaying the landmark information, we use java paging to enable the same numbers of landmark information on each page. Also you could find varieties of landmarks through turning pages, or process second search at current page.

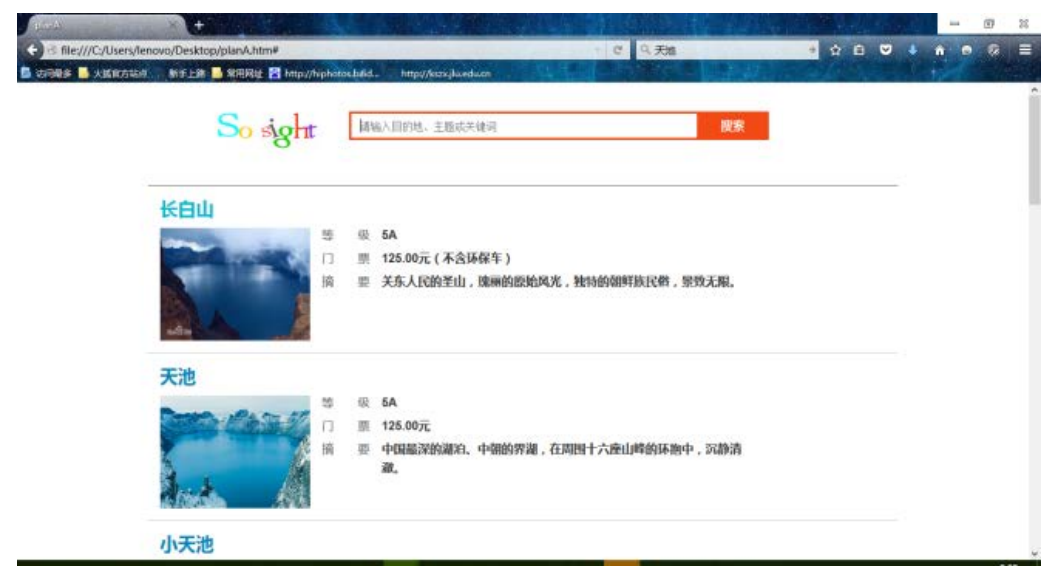

Figure 3 Result Display Interface

At the foundation of the code from PC, we complete the code of mobile phone. According to the reusability of the system, mobile terminal share some of the code with PC terminal. Also, PC and mobile terminal share the server, so we could search the pictures at the mobile terminals as well. The interface of search pictures on WeChat platform is as Figure 4.

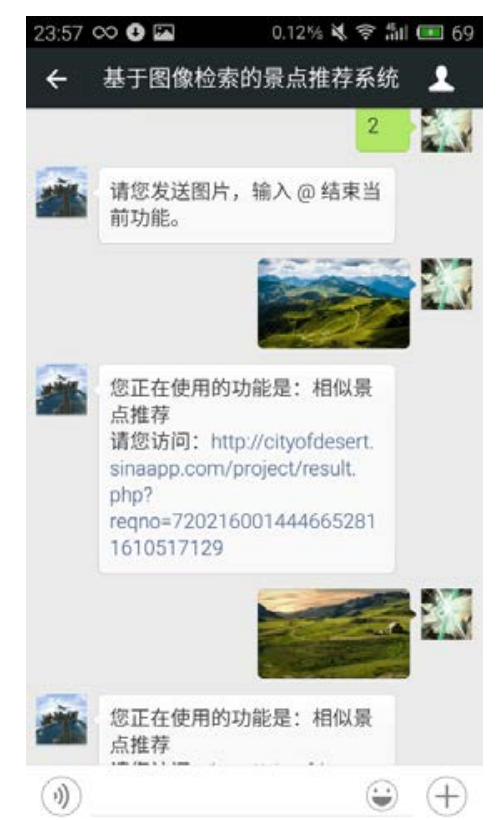

Figure 4 Retrieval pictures on WeChat

\subsection{Build Users' Evaluation Subsystem}

Users' evaluation subsystem could be divided into three parts: log in and exit, registration, and user evaluation. For user registration, the system could check if there has been same user through matching the registration information with the information in the database; if haven't, register successfully. Servlet program could write the user information, landmark information, evaluating time, and evaluation into the specific table in database. While other users make choice between these landmarks, the information could be used for reference. 


\section{Conclusion}

This paper illustrates the design and implementation of tourist landmarks recommending system which is based on multiple feature fusion and bidirectional matching image retrieval strategy. From requirement analysis, system integral design and system function implementation these three parts do this paper makes detailed presentation. B/S structure could satisfy the general requirement, and mature MVC tech with the stabilized, secure, advanced MySQL database constitutes the kernel tech support. Through this platform, users could recognize landmarks and search similar landmark and their dynamics according to personal requirement. Also users could make evaluations to their familiar landmarks. Compared to the traditional text-based retrieval, image-based input could present the users' demands and purposes more accurately and definitely, bringing great convenience to the travelers.

\section{Acknowledgment}

The corresponding author is Hongwei Zhao. The authors are grateful to the anonymous reviewers for their insightful comments which have certainly improved this paper. This work is supported by Plan for Scientific and Technology Development of Jilin Province (20140101184JC).

\section{Reference}

[1]Davis M, King S, Good N, et al. From context to content: leveraging context to infer media metadata.[C]// ACM multimedia.2004:188-195.

[2]Kennedy L S, Naaman M. Generating diverse and representative image search results for landmarks[C]//WWW.2008:297-306.

[3] Ren Y, Yu M, Wang X J, et al, Diversifying landmark image search results by learning interested views from community photos.[C]//WWW.2010:1289-1292.

[4]Ye J F. Image-Based Diversified Landmarks Search [D].Shanghai Jiao Tong University (2013).

[5]Zhu Y H. Mining and Augmented Reality for Web Landmark Photos [D]. Shanghai Jiao Tong University (2013) 